Herbert Schmidt

\title{
Fremde, ausländische, verlateinte, korrumpierte lateinische Wörter. Bezeichnungen für Fremdwörter im 16. Jahrhundert
}

\section{Fremdwort - Begriff und Ausdruck}

Die Geschichte des Begriffs ,Fremdwort", für deren Gesamtdarstellung sich bislang noch kein Historiograph gefunden hat, reicht im deutschsprachigen Raum zurück bis (mindestens) ins frühe 13. Jahrhundert und umfasst damit gut 800 Jahre. In jener Zeit werden erste Reflexe eines Bewusstwerdens dieses lexikalischen Sondertyps innerhalb des deutschen Wortschatzes fassbar: Wörter aus fremden Sprachen, die ins Deutsche entlehnt werden. Mit ihnen soll der Deutsche, so stellt Thomasin von Zerklære es ihm in seinem Welschen Gast frei, ruhig das einfarbjge Gewand seiner „tiusche“ mit bunten Streifen verschönern, da er dadurch „der spæhen worte harte vil“ lernen kann. ${ }^{1}$ Französische Modewörter aus der Sphäre der höfischen Kultur erfreuen sich in deutschen literarischen Texten des Hochmittelalters wachsender Beliebtheit, und dass dies bereits um die Mitte des 13. Jahrhunderts nicht nur registriert, sondern auch verhalten kritisch bewertet wurde, lässt sich aus persiflierenden Stellen wie der oft zitierten aus dem dritten Leich des Tannhäusers ${ }^{2}$ erschließen. Nur wenig später macht sich Wernher der Gärtner in seinem Helmbrecht die durch unangebrachten (und noch dazu falschen) Fremdwortgebrauch entstehenden Missverständnisse zur Erzielung komischer Effekte zunutze 3 : Wörter und Wendungen aus fremden Sprachen (oder auch nur

1 Der wälsche Gast des Thomasin von Zirclaria. V. 39-45: „daz ensprich ich dâ von nîht / daz mir missevalle iht / swer striffelt sîne tiusche wol / mit der welhsche sam er sol; wan dâ lernt ein tiusche man, / der niht welhische kan, / der spæhen worte harte vil“ (Hinweis bei Jones 1995: 4). Obwohl Thomasin den Gebrauch romanischer Fremdwörter in deutschen Texten empfiehlt, verzichtet er selber ausdrücklich darauf. V. 33-36: „Hie wil ich iuch wizzen lân, / swie wol ich welhische kan, / sô wil ich doch in mîn getiht / welhischer worte mischen niht."

2 Tannhäuser: Der winter ist zergangen, Strophe 7 (100): „Ein rifiere ich dâ gesach · / durch den fôres gieng ein bach · / ze tal über ein plâniure · / ich sleich ir nâch, unz ich si vant · die schœenen crêâtiure · bî dem fontâne sas diu clâre, diu süese von faitiure.“

3 Wernher der Gartenære: Helmbreht, V. 717-768 pass.

https://doi.org/10.1515/9783110698992-006 
fremden Mundarten), diese Erfahrung war unter den Zeitgenossen offenbar schon verbreitet, werden von den Einheimischen oft nicht oder falsch verstanden und erschweren die Kommunikation. Und wenn sich ungebildete, d. h. nicht lateinkundige Sprachteilhaber einmal an der Wiedergabe stark fremdworthaltiger (etwa juristischer) Verlautbarungen versuchen, dann entstehen leicht Verballhornungen wie doxer, lotari, bappeliert (für doctor, notari, appelliert), die der Gebildete, wie es der Freiburger Drucker Friedrich Riederer 200 Jahre später, am Ende des 15. Jahrhunderts tut, als „Barbarolexis“ bezeichnen muss. ${ }^{4}$ So war also das (vorwissenschaftliche) Konzept des Fremdworts mit vielen seiner integrierenden inhaltlichen Elemente - Herkunft aus einer Fremdsprache, hohes Sprachprestige, Verwendung überwiegend durch die sprachlichen Eliten, Schwerverständlichkeit und Gebrauchsschwierigkeit für Ungebildete - schon recht früh ausgebildet und hatte bereits jahrhundertelang sowohl gelehrte Reflexion wie leidenschaftliche Polemik erfahren, bevor der Ausdruck überhaupt entstanden war, mit dem wir es heute bezeichnen.

Der Ausdruck Fremdwort ist erst im frühen 19. Jahrhundert, also vor gut 200 Jahren, aufgekommen. Die neuere Forschung führt seit Weigand ${ }^{5}$ dieses Kompositum auf Jean Paul und das Jahr 1819, seit von Polenz ${ }^{6}$ auf Friedrich Ludwig Jahn (1816) und seit Kirkness ${ }^{7}$ auf Karl Christian Friedrich Krause (1815) zurück, wobei bis in die jüngste Zeit die „Erfindung" dieses Ausdrucks entweder einem oder mehreren der drei genannten Autoren zugeschrieben wird. ${ }^{8}$ Demgegenüber lässt sich heute - dank der immer zahlreicheren Digitalisate historischer Texte im Internet - die Verwendung des Kompositums Fremdwort durch den bereits erwähnten

4 Friedrich Riederer: Spiegel der waren Rhetoric, 1493, fol. XLIIIIb/XLVa: „Das ander laster wenn einer die wort gebrechenhafft vßdruckt Als sprech er. der doxer ist Atzolons vnd moses wirmer fürsprech gewesen gegen den frowen zun rüwernun vnd het durch ein lotari von der vrteil bappeliert So er sprechen sölt. Der doctor ist absolons vnd moyses widmeyer fürsprech gewesen gegen frowen zun rüwem vnd hett durch ein notari von der vrteil appelliert: Dis laster wenn einer die wort in einer frömbden sprach übel vßtruckt als so ein tutscher latinisch wort namlich lotari vnd bapaellieren gebrochen redt wirdt genannt Barbarolexis. " Zitiert nach Rössing-Hager (1992: 363) (mit den dort vorgenommenen Hervorhebungen durch Kursivierung. Im Original steht fol. XLVa allerdings nicht Barbarolexis, sondern Baralexis).

5 Weigand ( $\left.{ }^{5} 1909: 582\right)$.

6 von Polenz (1999: 265).

7 Kirkness (1975: 234f).

8 So etwa Jahn und Jean Paul (Wiegand 2001: 62, Heier 2012: 29), nur Krause (Paul ${ }^{10} 2002: 351$ ), nur Jean Paul (DWB Neub. IX 2006: 991), nur Jahn (Eisenberg 2012: 114) sowie Krause und Jahn (Kremer 2013: 196). 
Krause, aber auch durch Christian Moritz Pauli, Christian Hinrich Wolke u. a. bis ins Jahr 1811 zurückverfolgen.9

An älteren, bis zum Anfang des 19. Jahrhunderts gebräuchlichen Bezeichnungen für Fremdwörter nennt die neuere Literatur fremde Wörter ${ }^{10}$, ausländische Wörter ${ }^{12}$, undeutsche Wörter ${ }^{12}$, ausheimische Wörter ${ }^{13}$, Welschwörter ${ }^{14}$; deren Aufkommen wird meist vage in die Mitte des 17. Jahrhunderts datiert und mit dem sprachpuristischen Diskurs der Sprachgesellschaften in Verbindung gebracht ${ }^{15}$. Die von Rössing-Hager ${ }^{16}$ und Jones ${ }^{17}$ mitgeteilten deutschsprachigen Texte meist humanistischer Autoren, die bereits im späten 15. und im 16. Jahrhundert die Ubernahme von Fremdwörtern ins Deutsche kommentierten, machen allerdings klar, dass diese Datierung zu kurz greift: schon vor dem 17. Jahrhundert waren Fremdwörter ein Thema und mussten bezeichnet werden können, und diesem Zweck dienten damals auch einige der erwähnten Wendungen, aber nicht nur diese.

\section{Fremdwortreflexion im 16. Jahrhundert}

Im 16. Jahrhundert beginnen sich die Äußerungen und Stellungnahmen zu Fremdwörtern allmählich zu mehren, und erste lexikographische Zusammenstellungen von Fremdwörtern wurden geschaffen: zunächst noch in Form kürzerer Glossare in Handbüchern unterschiedlicher Thematik, dann aber auch, nämlich von Simon Roth in den siebziger Jahren des Jahrhunderts, in einem eigenständigen Teutschen Dictionarius, der heute als "erstes deutsches Fremdwörterbuch“ gehandelt wird. ${ }^{18}$ Eine Sammlung und Sichtung aller auffindbaren Textzeugnisse des 16. Jahrhunderts unter der Fragestellung, wie dort Fremdwörter bezeichnet

9 Näheres hierzu demnächst in Herbert Schmidt, Zur Entstehung des Ausdrucks Fremdwort im 19. Jahrhundert (Ms., noch unveröff.).

10 So Kluge $\left({ }^{9} 1921-^{21} 1975\right)$, Paul $\left({ }^{5} 1966-{ }^{9} 1992\right)$, Kirkness (1976: 230$)$, Duden Fremdwörterbuch ( $\left.1990-^{7} 2001\right)$, Kremer (2013, a. a. 0.)

11 Ebd.

12 von Polenz (1999, a. a. O.), Kremer (2013, a. a. O.)

13 Duden Fremdwörterbuch (72001: 524).

14 von Polenz (1999, a. a. O.), Kremer (2013, a. a. 0.)

15 Etwa bei Kluge ( $\left.{ }^{9} 1921{ }^{21} 1975\right)$, Bußmann (1990: 253), Duden Fremdwörterbuch ( $\left.{ }^{5} 1990: 9\right)$.

16 Rössing-Hager (1992).

17 Jones (1995).

18 Simon Roth: Ein Teutscher Dictionarius. Augsburg: Manger 1571 (VD16 R 3265). Dazu Kremer (2011) und Kremer (2013). 
werden und was damit über die Einstellung zu Fremdwörtern jeweils ausgesagt wird, erscheint umso wünschenswerter, als diese Vor- und Frühphase des Fremdwortdiskurses (im Gegensatz zu dessen Höhepunkten im 17. und besonders 19. Jahrhundert) von der Forschung bisher noch wenig beachtet wurde. Im Vergleich zur intensiven Erörterung der Fremdwortproblematik im 17. Jahrhundert handelt es sich bei den Stellungnahmen des 16. Jahrhunderts noch um sporadische, nicht im Zusammenhang einer strukturierten Debatte stehende Außerungen. ${ }^{19}$ Das erschwert das Auffinden dieser weit verstreuten Textstellen erheblich, und eine noch so extensive Konsultation der Quellen wird immer nur einen mehr oder weniger großen Ausschnitt aus dem Gesamtspektrum des Einschlägigen ans Licht bringen können. Die nachfolgend aufgeführten Fundstellen, ein rundes Dutzend, stammen nur zum kleineren Teil aus den erwähnten Anthologien von Rössing-Hager und Jones. Mehrheitlich sind sie entweder das Ergebnis gezielter Suche in thematisch einschlägigen Texten, in denen das Vorkommen von Fremdwortbezeichnungen erwartbar ist, oder das Resultat von Online-Recherchen in Digitalisaten zeitgenössischer Texte nach Ausdrücken, die aus späteren Zeiten als Fremdwortbezeichnungen bekannt oder grundsätzlich als solche denkbar sind. Bei einigen handelt es sich auch um reine Zufallsfunde. Abweichend vom differenzierteren modernen Fremdwortbegriff (der z. B. auch Lehnwortbildungen einschließt) werden hier als Objekte dieser Bezeichnungen schlicht deutsche Wörter verstanden, die aus fremden Sprachen stammen. Die verschiedenen Bezeichnungen werden in der chronologischen Reihenfolge ihres Auftretens vorgestellt.

\section{Fremdwortbezeichnungen im 16. Jahrhundert}

Unter den verschiedenen Bezeichnungen für Fremdwörter im 16. Jahrhundert findet sich kein einziges Kompositum (wie etwa das spätere Welschwort eines ist), sondern es handelt sich immer um die Verbindung des frühneuhochdeutschen Substantivs wort mit einem Adjektiv oder einem Partizipialattribut. Das Syntagma fremd(es) wort (das Adjektiv wird damals im Nom./Akk. Sg. meist unflektiert verwendet), aus dem dann später in einem wortschöpferischen Akt von eher bescheidener Originalität das Kompositum Fremdwort gebildet wurde, taucht gleich als frühestes und danach noch mehrfach auf, aber nur als eines unter vielen anderen. Eine echte Favoritenrolle dieser Wendung ist für den Untersuchungszeitraum noch nicht nachweisbar.

19 Jones (1995: 5). 


\section{1 fremd}

Das Syntagma fremd wort ist im Frühneuhochdeutschen (und noch lange darüber hinaus) grundsätzlich mehrdeutig, da fremd neben ,ausländisch, auswärtig, nicht einheimisch` auch ,unbekannt, nicht vertraut, neu' oder auch ,ungewöhnlich, auffallend, seltsam, wunderlich, wunderbar, sonderbar, unerhört, befremdlich ${ }^{20}$ bedeuten kann (und noch einiges mehr). Ein fremd wort kann also ebenso gut ein ,ausländisches, fremdsprachliches Wort' sein wie ein ,unbekanntes, nicht geläufiges Wort' oder ein ,seltsames, merkwürdiges, ungewöhnliches, befremdliches Wort' ${ }^{t}$ Wann genau in einem historischen Text, in dem dieses Syntagma vorkommt, die Rede von einem Fremdwort im heutigen Sinne ist, kann also nur aus dem jeweiligen Kontext erschlossen werden, und der ist nicht in jedem Fall eindeutig. So kann nur für einen Teil der nachfolgenden Fundstellen mit einiger Sicherheit angenommen werden, dass damit ein Wort aus einer Fremdsprache bezeichnet wird.

Den frühesten Beleg für das Syntagma fremd wort im 16. Jahrhundert liefert ein Werk Martin Luthers aus dem Jahr 1522.21 Der Autor äußert sich darin (fol. 80a) zur Aufeinanderfolge der beiden synonymen Ausdrücke „Abba pater“ im Neuen Testament (Gal. 4,6):

das hat villeicht sant Paulus auch gewölt / da er Abba / das hebreysch vnbekant frembd wort / zuuor an setzt / vnd darnach pater / das kriechisch bekant heymisch wort / die weil er vff kriechisch schreibt vnd den krtechen prediget.

Luther stellt hier in einem Parallelismus die Syntagmen das hebreysch vnbekant frembd wort und das kriechisch bekant heymisch wort einander gegenüber, sodass man einigermaßen sicher sein kann, dass frembd an dieser Stelle als Antonym $\mathrm{zu}$ heimisch verwendet wird und somit, ausländisch, fremdsprachlich, aus einer fremden Sprache stammend" bedeutet. Hier liegt also gleich ein Fall vor, wo man fnhd. frembd wort tatsächlich mit nhd. Fremdwort übersetzen kann.

Anders verhält es sich mit Textstellen wie der folgenden. Philipp Melanchthon interpretiert 1524 in seiner Auslegung des Johannes-Evangeliums ${ }^{22}$ die Selbstaussage Johannes des Täufers gegenüber den Pharisäern (Joh 1,23 mit den

20 Vgl. DWb IV.1.1 (1878): 125-128, DWb Neub. IX (2006): 979-985 (fremd) und 991 (Fremdwort). Vgl. auch Lexer III (1878): 500f. s. v. vremde, vremede; vrömde, vrömede.

21 Martin Luther: AVszlegung der Epistelen md Euangelien / die nach brauch der kirchen gelesen werden durch den Aduent / vnd dannenthyn vom Christag biß vff den Sontag nach Epiphanie [...] Basel 1522 (VD16 L 4551).

22 Verzaichnung vnd kurtzliche antzaigung in das Euangelium Joannis Philippi Melanchthons. [Augsburg: Grimm] 1524 (VD16 M 2485), fol. 17b. 
Worten von Jes 40,3: „Ich bin eine Stimme eines Predigers in der Wüste“), indem er zunächst den Ausdruck Stimme explaniert:

Vnnd zum ersten / nimbt er die zeligknuß seiner berieffung vnnd des worts / auß dem propheten Esaia darmit er antzaige / [...] das sein wort sey das wort gottes. Ich bin ain [...] rieffende stimm / oder ain verkünder / Ich bin ain stimm / nit das liecht. Item ain stimm / dann ich verkünd ain frembd wort / ich erdicht oder ler kain new wort.

Hier liegt wohl auf der Hand, dass frembd wort kaum die Bedeutung ,Wort aus einer Fremdsprache' haben kann, sondern eher ,merkwürdiges, seltsames, unerhörtes Wort' bedeuten dürfte (Wort metonymisch zu verstehen als ,Lehre, Botschaft').

Seit 1528 ist in einem oft nachgedruckten medizinischen Traktat mit dem Titel Kleine Wundartzney ${ }^{23}$ die Wendung „verlateinete und fremde wörter" alš Bezeichnung für Fremdwörter belegt; mit diesem Syntagma werden wir uns unten in Abschnitt 3.3 noch näher befassen. Die so bezeichneten (vor allem medizinischen) Fachwörter können (außer als verlateinet, was auch immer das bedeuten mag) ebensogut als fremdsprachlich wie als unbekannt oder auch als merkwürdig charakterisiert werden. Was genau der Autor hier gemeint hat, lässt sich nicht mit letzter Sicherheit bestimmen. Die Wahrscheinlichkeit ist am größten, dass hier in erster Linie von fremden i. s. v. fremdsprachlichen, aus einer fremden Sprache stammenden Wörtern gesprochen wird, doch es ist nicht ausgeschlossen, dass für den Autor an dieser Stelle eine der anderen Bedeutungen überwogen hat (oder auch die beiden anderen bewusst mitschwingen sollten).

Klare Fremdwortbezeichnungen wiederum liegen wohl an den folgenden Stellen vor, die aus Texten dreier anderer Autoren stammen: Caspar Hedio, Johannes Turmair (Aventinus) und Valentin Ickelsamer. Caspar Hedio gibt in der Chronica der Alten Christlichen Kirchen von $1530^{24}$ für die Wesensgleichheit von Gott Vater und Jesus Christus das griechische theologische Fachwort an:

Also wenig außgnomen / sind viler gmütter betrogen worden / wider die erkantnis der vetter in Nicea / die do gsagt vnd erkant haben / Man solt das vnbekant vnd frömbd wort Homousion in aller geschrift nit lassen bleiben in der außlegung des glaubens / diese haben ir gmeinschaft / mit der ketzer geselschaft vermaßgett.

Wie schon Luther das hebräische Wort $a b b a$ (s. o.) nennt Hedio griech. Homousion nicht nur ein „frembd wort“, sondern ein „vnbekant vnd frömbd wort“. Damit

23 S. u. Fußnote 31.

24 Caspar Hedio: Chronica der Altenn Christlichen kirchen. Straßburg: Georg Ulrich 1530 (VD 16 E 4286), fol. 85a. 
zieht er ein weiteres Adjektiv heran, das in Fremdwortbezeichnungen jener Zeit wiederholt auftritt (s. u. 3.4). Es bleibt hier allerdings offen, ob Hedio den griechischen Terminus als Fremdwort im heutigen Sinne (von fremder Herkunft, aber mehr oder weniger integriert und geläufig) oder eher als fremdsprachliches Zitatwort auffasst.

Aventinus und Ickelsamer lassen sich jeweils ausdrücklich über Fremdwörter im Deutschen aus, wodurch ihr Verständnis des Syntagmas frembd wort hier in aller wünschenswerten Eindeutigkeit feststeht. Aventinus beklagt in der Vorrede seiner Bayerischen Chronik (1533), dass viele lateinkundige Autoren seiner Zeit die deutsche Sprache über Gebühr nach lateinischem Vorbild ummodeln, und schreibt in dieser sprachkritischen Passage (auf die wir unten noch einmal zurückkommen werden):25

Es laut gar übel [...] wo man das teutsch vermischt mit frembden worten, verändert's auf ein frembde sprach.

Ickelsamer wiederum weist in seiner Teutschen Grammatica (um 1535) auf Fremdwörter hin, die im deutschen Wortschatz bereits verbreitet sind, und führt dafür auch einige Beispiele an: ${ }^{26}$

[D]ie telitschen [...] solten sich auch nit schämen etwa frembder wörter bedeütung zů lernen vnnd zůerfaren / dieweil sy auch deren vil in der teütschen sprach eben so gemain als die teütschen wörter selbs von allerlay dingen / gebrauchen / Dann welcher brauchet vnd redet nit Supplicatz / Citatz / Policey / Syndicus / Vocat? In der Artzney / Recept / purgatz / Clystier / von Kranckhaiten / Podagra / Colica Feber vnd des gleichen vil / Welches doch nit teütsche wort sein / sonder Lateinisch vnd Ghriechisch.

Es ist wohl kein Zufall, dass die von Ickelsamer aufgezählten Fremdwörter aus den Fachsprachen der Jurisprudenz und der Medizin stammen - erweisen sich doch genau diese beiden akademischen Fächer im 16. Jahrhundert geradezu als Einfallstore für (gelehrte) Fremdwörter in den deutschen Wortschatz. Anders als Aventinus vertritt Ickelsamer einen fremdworttoleranten Standpunkt und scheint die bereits aufgekommenen Fremdwörter als unabdingbar und ,eingebürgert' zu akzeptieren. Auffällig ist auch, dass er die frembden wörter ungeachtet ihrer in den meisten Fällen erkennbaren morphologischen Integriertheit kurzerhand als lateinische und griechische Wörter bezeichnet (s. u. 3.7).

25 Johannes Turmair's genannt Aventinus Bayerische Chronik. Erster Band. Erste Hälfte. 1882; 6. Vgl. unten Abschnitt 3.6.

26 Valentin Ickelsamer: Teutsche Grammatica. [Augsburg, um 1535] (VD16 I 26), fol. D4b. 
Während sich bei Hedio und Ickelsamer für das Syntagma frembd wort die Bedeutung ,ausländisches, aus einer Fremdsprache stammendes Wort ${ }^{\star}$ mehr oder weniger zwingend aus den beigegebenen fremdsprachigen Beispielwörtern ergibt, erhellt dies im nachfolgenden letzten Fall, wie schon bei Aventinus, zweifelsfrei aus dem Kontext. Hier wird die Frage erörtert, warum und wie Fremdwörter ins Deutsche gekommen sind. Das Zitat stammt aus Simon Roths Vorrede zum Teutschen Dictionarius von 1571, und es stellt bezeichnenderweise die einzige Stelle dar, an der in diesem ,ersten deutschen Fremdwörterbuch" die Wendung frembd wort auftritt:27

Darumb halt kain wunder / das schon vil Gelerten wöllen / das die Teutsche sprach / den maisten thell ein gesamlete sprach / auß allerley sprachen sey. Vnd geben des solche vrsach für / Dieweil die Teutschen etwa gar ein Kriegpar volck gewesen / ja sonst schier anders nichts gethan / dann dem selben obgelegen / Darumb sie dann von andern Nationen auffgemanet / vnnd zu gehilffen seind bestelt worden / vnnd also in frembde Landt kriegen nachzogen / vnnd auß denselben nit allein frembde sitten / geberdt vnd kleider / sonder auch vil frembde wort haim in jr Landt gebracht.

An der exponiertesten Stelle des Dictionarius, im Titel, beschreibt der Autor naheliegenderweise den Wortschatz, der in diesem Wörterbuch gesammelt und erläutert wird. Hier spielt, wie gesagt, das Syntagma frembd wort keine Rolle. Stattdessen tauchen dort andere Adjektive auf, die im Weiteren noch zu betrachten sein werden (s. u. 3.4 und 3.5).

\section{2 ausländisch}

Das Syntagma außlendige wörtter findet sich bereits 1523 in einem Basler Druck von Luthers Newem Testament ${ }^{28}$, bezeichnet dort aber strenggenommen nicht das, was wir heute unter Fremdwörtern verstehen. Der Drucker Adam Petri bezieht sich damit vielmehr auf rund 200 mitteldeutsche Luther-Wörter, die er zum besseren Verständnis für seine einheimischen Leser in einem beigegebenen Glossar mit oberdeutschen Entsprechungen versieht. ${ }^{29}$ Es handelt sich hier also

27 Simon Roth: Ein Teutscher Dictionarius. 1571. Vorrede, fol. A6a.

28 [Martin Luther:] DAs neuw Testament recht grüntlich teutscht. Basel: Adam Petri 1523(VD16 B 4325).

29 Auf dem Titelblatt heißt es bereits (fol. A1a): „Die außlendigen wörtter / auff vnser teutsch angezeygt." Das Glossar folgt auf drei Seiten ab fol. A3b und wird von Petri eingeleitet: „Lieber Christlicher Leser / So ich gemerckt hab / das nitt yederman verston mag ettliche wörtter im yetzt gründtlichen verteutschten neuwen testament / [...] hab ich lassen die selbigen auff vnser hoch teutsch außlegen vnd ordenlich in ein klein register wie du hie sihest / fleißlich verordnet.“ 
um Wörter nicht aus einer Fremdsprache, sondern aus einer anderen deutschen Mundart, und bei dem in außlendig anklingenden "Ausland" lediglich um eine andere deutsche Dialektregion.

Auf „echte", aus Fremdsprachen entlehnte Fremdwörter dagegen beziehen sich knapp fünfzig Jahre später zwei Stellen in dem bereits erwähnten Teutschen Dictionarius von $1571^{30}$, wo der Autor Simon Roth außlendische wort erwähnt. In der Vorrede heißt es am Ende eines längeren Exkurses über den entlehnungsaffinen Nationalcharakter der Deutschen (fol. A6b):

Nun wol solches [...] etwas zu weitschwaiffig geacht möcht werden / hab ich es doch wöllen melden / damit man wiß / auß was vrsach souil Barbarische oder außlendische wort in Teutsche sprach kommen sind.

Und im Wörterbuchteil wird das Lemma Nunn bzw. Nonna charakterisiert als „Ein außlendisch wörtel / das in die Lateinisch sprach nit gehört“ (fol. L3a).

Die Kombination mit dem Adjektiv barbarisch im ersten Textausschnitt verleiht außlendisch für heutige Ohren ein eher pejoratives Gepräge. Es ist aber zu bedenken, dass der Autor dieses Textes ein humanistisch gebildeter Lateinschulmeister ist, der seine lateinisch- oder griechischstämmigen Fremdwörter automatisch so versteht, wie deren Etyma in der klassischen Antike verstanden wurden. Die Grundbedeutung von lat. barbarus aber ist ,ausländisch, fremd', ohne negative Wertung (erst in metonymischer Verwendung wird daraus, roh, unkultiviert, ungeschliffen, wild'), sodass die Wendung Barbarische oder außlendische wort hier als synonyme Doppelformel anzusprechen ist. An der zweiten Stelle dient außlendisch wörtel offenbar zur Kennzeichnung eines Ausdrucks, der im Gegensatz zur großen Mehrheit der Roth-Lemmata nicht aus dem Lateinischen, sondern aus einer anderen (lebenden?) Fremdsprache stammt.

\section{3 verlateinet}

Seit 1528 ist im Wortfeld der Fremdwortbezeichnungen das Adjektiv verlateinet belegt. In Otto Braunfels' deutscher Ubersetzung von Lanfranks Kleiner Wundartzney ${ }^{31}$ wird schon auf dem Titelblatt unter dem Titelholzschnitt auf ein Fremdwortglossar am Ende des Werks hingewiesen: „Der verlateineten vnd frembden wörter außlegung sůch am end“. Dort, fol. F3b, werden dann unter der Uberschrift „Auß-

30 S. o. Fußnote 18; Näheres s. u. Abschnitte 3.4 und 3.6.

31 Kleine Wundartzney des hochberümptenn Lanfranci [...] / durch Othonem Brunfels verteutscht. [Augsburg: Heinrich Steiner 1528] (VD16 L 245). 
legung der verlateineten vnd frembden wörter " insgesamt 34 überwiegend medizinische und mehrheitlich lateinischstämmige Fachwörter samt Eindeutschung aufgelistet:

\author{
Abstergiern abtrïcknen \\ Accidens zůfall \\ Apostemirn schweren \\ Curiern heylen \\ Cicatrisiern vergentzen \\ Confortiem stercken \\ Coliern seygen \\ Consolidiern gantz machen \\ Collyrium augen salb \\ Compositum zủsamen gethan \\ Cauteriziern brennen \\ Constringiem stopffen \\ Corrodiern etzen \\ Diet regiment \\ Euacuirn außreynigen \\ Embrociem reüchen oben $\mathrm{ab}$. \\ Fomentiern bähen vnden auf.
}

\author{
Incarniern zů fleisch bringen \\ Mundificlern reynigen \\ Maturirn zeytigen. \\ Opilieren stopffen \\ Puluerisiern puluern. \\ Procediern faren \\ Principia anfang \\ Pacient siech / kranck \\ Putrificiern feülen \\ Repercutirn hinderschlagen \\ Resoluiern zerlassen \\ Simplicia eynfachen. \\ Suppositoria ars zapffen \\ Trocisci seind kügelin \\ Temperiern einmachen \\ Vngent salb \\ Ventosen schrepff hörnlein.
}

In den späteren Ausgaben dieses Werks (Straßburg 1528 [2], Erfurt 1529, Zwickau 1529, Erfurt 1530 und 1534 ${ }^{32}$ ) bleibt die Uberschrift des Glossars zunächst gleich ${ }^{33}$, doch mit der Frankfurter Ausgabe von $1552^{34}$ wird sie ersetzt durch eine andere, die in den weiteren Ausgaben bis zum Ende des Jahrhunderts (Magdeburg 1585, Frankfurt 15945) beibehalten wird. Dort heißt es nun: „Verteutschung Latinischer Wörter inn diesem Büchlein“ (Frankfurt 1552, fol. 29a) bzW. „Verteudschung der Lateinischen Wörter in diesem Buch“ (Magdeburg 1585, fol. A2b). Das hier zudem mit frembd (s. o. 3.1) kombinierte Adjektiv verlateinet wird also in der zweiten Jahrhunderthälfte zugunsten des unmittelbar die Herkunftssprache bezeichnenden lateinisch (s. u. 3.7) aufgegeben; vielleicht ein Indiz dafür, dass es den Druckern der späteren Ausgaben nicht mehr geläufig war oder sogar miss- oder unverständlich erschien. Auch heute bereitet im vorliegenden Kontext sein Verständnis eher Schwierigkeiten: verlateinet kann nur ,ins Lateinische übersetzt/übertragen“ be-

32 Straßburg: Christian Egenolph 1528 (VD16 L 247); Straßburg: Johann Schott 1528 (VD16 L 248); Erfurt: Melchior Sachs 1529 (VD16 L 249); Zwickau: Gabriel Kantz 1529 (VD16 L 253); Erfurt: Melchior Sachs 1530 und 1534 (VD16 L 254 und L. 255).

33 Der Hinweis auf dem Titelblatt dagegen verschwindet schon in den ersten Nachdrucken.

34 Frankfurt am Main: Hermann Gülfferich 1552 (VD16 L 256).

35 Magdeburg: Johann Francke 1585(VD16 L 260); Frankfurt am Main: Peter Kopf, Johannes Sauer 1594 (VD16 L 261). 
deuten. ${ }^{36}$ Inwiefern lässt sich das aber von den aufgeführten Fremdwörtern sagen? Sie stammen so gut wie alle direkt aus dem Lateinischen und wurden wohl kaum aus zugrunde liegenden deutschen Wörtern (im Sinne von Lehnwortbildungen) latinisiert. Einige dieser lateinischen Wörter allerdings gehen ihrerseits aufs Griechische zurück, wie etwa lat. diaeta, mlat. dieta (das dem hier an 14. Stelle auftretenden Fremdwort Diet zugrunde liegt) auf gleichbedeutend griech. diaita ,vom Arzt vorgeschriebene Lebensweise ${ }^{437}$. Für diese Wörter (auch Apostemirn und Collyrium gehören wohl dazu) gilt also in der Tat, dass sie einst (nämlich aus dem Griechischen) ins Lateinische übertragen worden sind. Aber das ist eine Aussage über die Etymologie des lateinischen Ausgangsworts, die an dieser Stelle nicht unbedingt notwendig erscheint. Die Uberschrift kündigt eine Liste „der verlateineten vnd frembden wörter" an, und auch diese Doppelformel wirft Probleme auf: soll der Leser unter den verlateineten Wörtern wirklich diejenigen verstehen, die übers Lateinische weiter aufs Griechische zurückgehen, und unter den frembden Wörtern die übrigen, die ursprünglich aus dem Lateinischen stammen? Das würde bedeuten, dass die beiden Ausdrücke hier nicht als Synonyme verstanden, sondern komplementär gebraucht werden, sodass für jedes Fremdwort entschieden werden muss, ob es nun verlateinet oder frembd ist. Die meisten zeitgenössischen Leser dürften von dieser Aufgabe überfordert gewesen sein.

Die Fremdwortbezeichnung verlateinet taucht noch in einem weiteren naturwissenschaftlichen, nämlich alchimistischen Text des 16. Jahrhunderts auf: Das anonym erschienene, Georg Agricola zugeschriebene Werk Rechter Gebrauch der Alchimei (1531) ${ }^{38}$ weist ebenfalls schon auf seinem Titelblatt auf das (sehr kurze) Glossar der „Alchimistischen verlateineten wörtter" hin, das dann auf fol. 1b folgt:

I Etlich verlateinet Alchimistische wörter.

Calx puluer

Calcinirn zů puluer oder kalck machen.

Corpus / ein iedes metal oder Materi.

Soluirn / zertreiben / zerscheyden.
Preparim bereyten.

Purgirn reynigen.

Recipe oder Ri / heyßt / Nim.

Die späteren, ausnahmslos in Frankfurt am Main erschienenen Ausgaben (um 1535, 1540, 1549, 1566 und 1574 ${ }^{39}$ ) erweitern das Glossar ab 1566 zwar geringfü-

36 DWB XII.1 (1956): 737 s. v. verlateinen: ,ins lateinische übersetzen, ex lingua alia in latinam transferre.

37 Deutsches Fremdwörterbuch (Neubearb.) IV (1999): 520.

38 [Georg Agricola?:] Rechter Gebrauch der Alchimei / Mitt vil bißher verborgenen / nutzbaren vnnd lustigen Künsten / [...] ๆ Der Alchimistischen verlateineten wörtter außlegung. [...] [Frankfurt am Main: Christian Egenolff d. Ä. 1531] (VD 16 R 492).

39 VD16 R 494 / R 497 / R 498 / R 500 / R 501. 
gig um zwei weitere Lemmata ${ }^{40}$, ändern an seiner Ubberschrift aber nichts. Das darin enthaltene Attribut alchimistisch macht die Zugehörigkeit der aufgelisteten Wörter zum entsprechenden Fachwortschatz klar, der Ausdruck verlateinet aber bleibt hier mindestens so problematisch wie in der Wundartzney (zumal hier die meisten Wörter nur aufs Lateinische, nicht aber aufs Griechische zurückführen). Vielleicht auch deshalb ist er im Zusammenhang mit Fremdwortbezeichnungen im 16. Jahrhundert sonst nicht mehr nachweisbar.

\section{4 unbekannt}

Der vollständige Titel von Simon Roths Wörterbuch von 1571 lautet (Titelblatt, fol. A1a):

Ein Teutscher Dictionarius / das ist ein außleger schwerer / nnbekanter Teutscher / Griechischer / Lateinischer / Hebraischer / Wälscher vnd Frantzösischer / auch andrer Nationen wörter / so mit der weil inn Teutsche sprach kommen seind / vnd offt mancherley jrung bringen: hin und wider auß manicherley geschrifften / vnd gemainer Red zusamen gelesen / außgelegt / vnd also allen Teutschen / sonderlich aber denen so zu Schreibereien kommen / vnd Ampts verwaltung haben / aber des Lateins vnerfarn seind / $\mathrm{zu}$ gutem publiciert.

Hier wird einigermaßen ausführlich Auskunft gegeben über die Quellen und Adressaten des Wörterbuchs, vor allem aber über den darin erklärten (,ausgelegten') Wortschatz: schwere und unbekannte Wörter, die oft zu Missverständnissen (jrrung) führen, und zwar nicht nur solche aus fremden Sprachen, sondern auch (native) deutsche Wörter. ${ }^{41}$ Die Fremdwörter werden wieder, wie bei Ickelsamer, schlicht als griechische oder lateinische Wörter bezeichnet, dazu kommen hier noch hebräische, welsche - das bedeutet in diesem Kontext: italienische -, französische sowie Wörter „andrer Nationen“. Doch wichtiger als das, was hier über die Gebersprachen gesagt wird, ist vor der Hand die auch bei Luther und Hedio vorkommende Charakterisierung als "unbekannt". Damit wird ein Aspekt angesprochen, den viele Sprachbenutzer (auch heute noch, wenn auch wohl in geringerem Maße als damals) mit Fremdwörtern in Verbindung bringen. Als allei-

40 "Coagulirn / schmeidig machen" und "Reducirn / widerbringen".

41 Dass auch erklärungsbedürftige deutsche Wörter im Dictionarius behandelt werden, scheint dessen typologische Zuordnung zu den Fremdwörterbüchern in Frage zu stellen. Doch ist die Anzahl der deutschen Lemmata verschwindend gering; Roth betrachtet von allen rund 2.450 Lemmata elgentlich nur Mar ,Ross' als nativ deutsch, allenfalls noch Voit "Beschützer'. (Darüber hinaus gibt es noch weitere 20 Lemmata, die heute als nicht-entlehnt gelten, die Roth aber fälschlich auf fremdsprachliche Etyma zurlickführt, doch kommt es hier ja nur auf Roths eigene Einschätzung an.) Vgl. Kremer (2013: 113-115). 
niges Merkmal dieses Worttyps ist er für die Autoren des 16. Jahrhunderts aber zur Kennzeichnung offenbar nicht ausreichend: Luther und Hedio sprechen jeweils von einem „vnbekant (vnd) frembd wort“, und Roth kombiniert unbekannt mit „schwer".

\section{5 schwer}

In allen derzeit bekannten Textstellen mit Fremdwortbezeichnungen aus dem 16. Jahrhundert taucht das Adjektiv schwer nur einmal auf, nämlich hier im Titel von Simon Roths Dictionarius. Auch dies ist eine typische Eigenschaft von Fremdwörtern: ihre Verwendung bietet Schwierigkeiten, sie sind schwer zu schreiben, auszusprechen, zu verstehen, richtig zu gebrauchen - besonders für die nicht lateinkundigen Sprachteilhaber, an die Roth als Adressaten seines Wörterbuchs vor allem denkt. Trotzdem wird auf dieses grundlegende Merkmal bei der Fremdwortbezeichnung zu seiner Zeit anscheinend nicht öfter abgehoben. Eine Pointe der metalexikographischen Forschungsgeschichte ist es, dass sich die moderne Typisierung des Dictionarius der ursprünglichen Charakterisierung durch den Autor inzwischen wieder angenähert hat. In ihren neuen Dictionarius-Studien diskutiert Anette Kremer die Berechtigung der traditionellen Einstufung als (erstes) Fremdwörterbuch und gelangt dabei, mit Blick auf den aus der angelsächsischen Lexikographie bekannten Wörterbuchtyp des hard word dictionary, zu einer differenzierteren Beschreibung: der Teutsche Dictionarius sei „ein Vorläufer heutiger Fremdwörterbücher [... ], angelegt als Wörterbuch der schweren Wörter““42

\section{6 korrumpiert/zerbrochen}

Eine der im 16. Jahrhundert auffälligsten Definitionen von Fremdwörtern ist die, sie seien mehrheitlich ,lateinisch / wiewol sie in Teutsch corrumpirt werden". Diese Wendung findet sich zum ersten Mal in dem 1536 erschienenen juristischen Handbuch Gerichtlicher Proceß $\beta$ von Justinus Göbler ${ }^{43}$, das breit rezipiert wurde und bis in die siebziger Jahre des Jahrhunderts noch sieben weitere Auflagen er-

42 Kremer (2013: 204). So auch bereits Kremer (2011: 215-218).

43 Justinus Göbler: GErichtlicher Proceß / auß grund der Rechten vnd gemeyner übung / zum fleissigsten in drei theyl verfasset. Das Erst Theyl / Nach erklerung aller vnd leder sonderen vnd eygenen wörter / so in den Rechten vnd Gerichten gebraucht werden / Dle Termin des Gerichtlichen Proceß / wie die nach ordenung der Rechten zuhalten seien / [...]. Getruckt zu Franckfurt am Mayn / Bei Christian Egenolff. [1536] (VD16 G 2296). 
lebte. ${ }^{44}$ Göbler fügt ins 2. Kapitel seines Handbuchs ein Glossar ein, in dem er die wichtigsten Fachtermini des Gerichtswesens auflistet: „Erklerung deren sondern vnnd eygenen wörter / so inn dem Gerichtlichenn Proceß / vnd sunst im Rechten gebraucht werden / wie die im Latein vnd Teutschen zuuerstehen seien" (fol. 3b). Dieses Glossar ist dreigeteilt und behandelt in den ersten beiden Teilen die Bezeichnungen für das am Gericht agierende Personal und die dort verwendeten Schriftstücke. Die Lemmata sind hier durchweg lateinische Substantive, die in ihren lateinischen Flexionsformen (im Nom. Sg. oder Plur.) angesetzt werden, die Interpretamente sind deutsch. Im dritten Teil des Glossars werden dann die Bezeichnungen der verschiedenen Gerichtshandlungen erklärt, und dabei geht es nun um (insgesamt 495) Verben, die aus dem Lateinischen stammen, aber mittels des Lehnsuffixes $-i(e) r(e) n$ eingedeutscht worden sind:

Nun ist noch übrig das dritteyl / nemlich die besondere vnd eygne wörter der handlungen / Actuum, zuerkleren (...) Nun sein dieselbigen der mehrerteyls Lateinisch / wiewol sie in Teutsch corrumpirt werden. ${ }^{46}$

Dieser Teil des Glossars beginnt mit den Lemmata Citim, Comparim, Constituirm, Substituirn, Reuocirn, Ratificirn und endet zweieinhalb Seiten weiter mit Deponirn, Iustificirn, Euincirn, Succedirn, Inuentiren und Qualificirn.

Inwiefern sind diese Lemmata nun „lateinisch“ und „im Deutschen korrumpiert"? Thnen allen liegen lateinische Verben aus unterschiedlichen Konjugationsklassen zugrunde: citare, comparare, constituere, substituere, revocare, ratificare usw. In dieser Hinsicht, was ihre Herkunft betrifft, sind die Lemmata des Glossars mithin lateinisch. Im Zuge ihrer Verwendung als Fremdwörter der deutschen Rechtssprache wurden sie allerdings, anders als die zuvor aufgezählten Substantive, flexionsmorphologisch ans Deutsche angeglichen, indem an die Stelle der alten lateinischen Infinitivendungen -are und -ere das deutsch flektierbare Lehnsuffix -ieren getreten ist. Diese Angleichung ans deutsche Flexionssystem, die sich positiv auch als Integration beschreiben ließe, wird hier nun dezidiert negativ als „Korruption“ bezeichnet: es handele sich um (eigentlich) lateinische Verben, die als Fremdwörter „in Teutsch corrumpirt“, also verderbt werden. Diese stark wertende Wortwahl gibt im Hinblick auf den Autor Anlass zu Vermutungen. Es könnte sich um jemanden handeln, für den Latein einen deutlich höheren Status hat als das Deutsche, und der jede morphologische Veränderung eines

44 Frankfurt am Main: Christian Egenolff 1538, 1542, 1549, 1555, 1562, 1567, 1578 (VD16 G 22972303).

45 Seit der Ausgabe von 1562 ist die Liste dieser Verben um 13 weitere auf 62 vermehrt.

46 Göbler 1536, fol. 7a. 
lateinischen Worts im Interesse seiner Integration ins Deutsche als sprachfrevelnde Entwertung, Herabminderung, Beschädigung empfindet. Oder es geht um jemanden, der sprachpuristische Ansichten vertritt und, ohne der einen oder der anderen Sprache einen höheren Wert beizumessen, einfach die Verwendung von Mischformen wie diesen aus teils lateinischen, teils deutschen Bestandteilen gebildeten Fremdwörtern ablehnt, der also jede Sprache rein und unvermischt und klar voneinander getrennt verwenden will.

Göbler scheint die Wendung ,in Teutsch corrumpirt" mit Bezug auf Fremdwörter als erster gebraucht zu haben. Ob er die dahinterstehende Vorstellung auch selbst aufgebracht hat, erscheint zweifelhaft. Kehren wir noch einmal zu der drei Jahre früher, 1533, verfassten Vorrede von Aventinus' Bayerischer Chronik ${ }^{47}$ zurück. Der Humanist begründet dort, warum er die deutsche Ubersetzung seiner ursprünglich lateinisch geschriebenen Chronik in einer alltäglichen, allgemeinverständlichen Sprache gehalten hat, und spricht sich bei dieser Gelegenheit gegen die Gewohnheit seiner lateinkundigen Zeitgenossen aus, ihre deutschen Texte nach lateinischem Muster zu ,verbiegen“ und zu „verkrümmen“ und mit „zerbrochenen lateinischen Wörtern" zu verfälschen:

[...] in dieser verteutschung brauch ich mich des alten lautern gewönlichen iederman verstendigen teutsches; dan unser redner und schreiber, voraus so auch latein künnen, biegen, krümpen unser sprach in reden, in schreiben, vermengens, felschens mit zerbrochen lateinischen worten, machens mit grossen umbschwaifen unverstendig ziehens gar von iret auf die lateinisch art mit schreiben und reden, das doch nit sein sol, wan ein ietliche sprach hat ir aigne breuch und besunder aigenschaft.

Es laut gar übel und man haist es kuchenlatein, so man latein redt nach ausweisung der teutschen zungen: also gleichermaß laut's übel bei solcher sach erfarnen, wo man das teutsch vermischt mit frembden worten, verändert's auf ein frembde sprach, demnach's zerbrochen und unverstendig wirt. (5/6)

Hier taucht die Partizipialform zerbrochen gleich zweimal auf: das Deutsche wird unzulässig „mit zerbrochen lateinischen worten“, also Fremdwörtern, verfälscht, und so, "vermischt mit frembden worten“, wird es „zerbrochen und unverstendig“. Aventinus, der humanistisch gebildete versierte Lateiner, vertritt hier einen entschieden sprachpuristischen Standpunkt und wendet sich gegen die Verwendung lateinischstämmiger Fremdwörter in deutschen Texten; sowohl diese Fremdwörter als auch die damit versetzten Texte klassifiziert er abwertend als „zerbrochen“. Der zunächst etwas merkwürdig erscheinende Ausdruck zerbrechen ist natürlich die etymologisierende, wörtliche deutsche Entsprechung von

47 Vgl. oben Fußnote 25. Die Chronik erschien erst 1556 im Druck, wurde aber bereits in den Jahren 1526-1533 verfasst. 
lat. corrumpere, das wir heute in diesem Kontext eher mit verderben übersetzen würden: Aventinus polemisiert hier gegen Fremdwörter als „verderbte lateinische Wörter" und gegen das latinisierte Deutsch als „verderbte und unverständliche“ Sprache. Damit drückt er sich im Grunde genauso aus wie Göbler später mit seinen ,lateinischen, aber im Deutschen korrumpierten Wörtern".

Ob Göbler die zu seiner Zeit noch ungedruckte Bayerische Chronik des Aventinus mit ihrer Vorrede gekannt und seine Fremdwortbezeichnung von dort übernommen hat, steht dahin. Zumindest belegt die Aventinus-Stelle, dass es zu dieser Zeit in humanistischen Gelehrtenkreisen (in welchem Ausmaß auch immer) eine Haltung gegeben hat, die den Fremdwortgebrauch ablehnt und Fremdwörter solchermaßen, als korrumpierte/zerbrochene lateinische Wörter, auffasst und bezeichnet.

Göblers juristisches Fachglossar wurde im 16. Jahrhundert in Werken ähnlicher Thematik immer wieder aufgegriffen und nachgedruckt, sowohl als Ganzes wie mit einzelnen seiner Worterklärungen. Dabei wurde auch die Redeweise von den korrumpierten Wörtern mehrfach mitübernommen. In einem ab 1538 anonym erscheinenden, verbreiteten Handbuch des Notariatswesens mit dem Titel Notariat vnnd Teutsche Rhetori ${ }^{48}$ taucht das komplette Glossar samt der Binnenüberschrift mit der einschlägigen Fremdwortbezeichnung seit der Ausgabe von $1561^{49}$ auf, sie lautet hier (fol, 15b):

Besondere vnd eygene wörter der handlungen / Actuum, seind der mehrertheyls Lateinisch / wiewol sie in Teutsch Corrumpiert werden / wie folget.

Auch in der verbreiteten Synonymik von Leonhard Schwartzenbach ${ }^{50}$ findet sich seit deren 1564 erschienenen dritten Auflage diese Wendung, und hier sogar schon im Titel des Werks (fol. Ala):

SYNONYMA. Formular, Wie man ainerley rede vnd mainung / mit andern mehr worten / auff mancherley art vnd weise / zierlich reden / schreiben / vnd außsprechen sol. Item /

48 Notariat vnnd Teutsche Rhetoric. Frankfurt am Main: Christian Egenolff 1538 (VD16 N 1870).

49 Notariat/ $m d$ Teutsche Rhetoric. Frankfurt am Main: Chr. Egenolffs Erben 1561 (VD16 N 1874). Glossar hier fol. 12a-16b. Weitere Ausgaben (mit Glossar) Frankfurt am Main bei Egenolffs Erben 1565, 1571, 1578 (VD16 N 1875, VD16 ZV 20783 / N 1876, VD16 N 1877).

50 Leonhard Schwartzenbach: Synonyma. Formular, Wie man ainerley rede vnd mainung / mit andern mehr worten / auff mancherley art vnd weise / zierlich reden / schreiben / vnd außsprechen sol. Frankfurt am Main: Feyerabend und Hüter 1564 (VD16 S 4677). Die ersten beiden Auflagen erschienen 1554 und 1556 (beide in Nürnberg bei Georg Merckel (Claes 1977: 118, Nr. 451 und 122, Nr. 466)), spätere Ausgaben 1571 und 1580 (Frankfurt am Main bel Hieronymus bzw. Sigmund Feyerabend, VD16 S 4678 und 4679). Vgl. dazu Haß (1986) mit faksimiliertem Nachdruck der Ausgabe 1564; Müller (2001: 252-258). 
Bericht vnd Außlegung etlich Lateinischer wörter / so täglich inn vnd ausserhalb Gerichts gebraucht / vnd zum theil im Teutschen corrumpiert werden. Auch vnterschied derselben / daneben vermeldet vnd angezeigt. Für die jungen noch vngeübten Schreiber gestellet. ${ }^{\text {11 }}$

Schwartzenbach löst die dreigeteilte Struktur des Göbler'schen Glossars komplett auf und verteilt dessen Lemmata so, wie sie semantisch passen, unter die Synonyme seiner rund 1.100 alphabetisch geordneten eigenen Lemmata. Die Interpretamente der Göbler-Lemmata aber übernimmt er so wortgetreu aus seiner Vorlage, dass deren Verwendung außer allem Zweifel steht. ${ }^{52}$ Im Anschluss an die Wörterbuchstrecke folgt dann (ebenfalls seit der 3. Auflage 1564) auf dem letzten Blatt noch ein zweiseitiges Register der in den Synonyma vorkommenden Fremdwörter (vgl. die Abb. 1 und Abb. 2), und dessen Überschrift lautet bezeichnenderweise:

Die Corrumpierte Lateinische Wort / davon anfangs gemeldet / vnd bey disen Synonymis verteutscht vnd außgelegt / sind dise (fol, 101a).

Das Register führt insgesamt 160 Fremdwörter (ganz überwiegend der Rechtssprache) auf, von denen im Werk 43 als Lemmata angesetzt, 117 als Synonyme nativ deutscher Lemmata genannt werden. Nach den typographischen Gepflogenheiten der Zeit sind die Fremdwörter meist, aber nicht immer in Antiqua (statt der Fraktur-Normaltype) gesetzt. ${ }^{53}$ Zählt man die in den Synonyma tatsächlich auftretenden Fremdwörter genau aus, ergibt sich eine höhere Anzahl, nämlich insgesamt 209; rund 65 davon sind im Register nicht erfasst. Dieses Fremdwortregister ist ein klarer Beleg dafür, dass dem Autor Schwartzenbach, sieben Jahre vor Simon Roth, der lexikalische Sonderstatus des deutschen Fremdwortschatzes bereits bewusstgeworden ist. Zwar gibt er durch die Übernahme der pejorativen

51 Ähnlich nochmals in der Widmungsvorrede fol. A2b: „Hab ich mit so viel weniger reuwe / diß Formular (...) an viel enden verneuwert / mit zuthuung etlich Lateinischer wörter / so im Teutschen corrumpiert / vnd täglichs in vnd ausserhalb Gerichts gebraucht werden / auff daß ein junger Schreiber auch dieselben (...) desto leichter verstehen möge."

52 Die 49 Lemmata der Göbler-Strecke Citim - Qualificim greift Schwartzenbach zudem so vollständig auf (es fehlt kein einziges), und die 13 seit der 6. Auflage des Gerichtlichen Proceß von 1562 hinzugekommenen Lemmata (vgl. oben Fußnote 45) fehlen bei Schwartzenbach so eindeutig (11 fehlen ganz, bei den übrigen zwei haben die Interpretamente einen völlig anderen Wortlaut als bei Göbler), dass sich daraus die benutzte Göbler-Ausgabe auf eine der ersten fünf Auflagen (1536-1555) eingrenzen lässt. Haß dagegen geht davon aus, Schwartzenbach habe für die dritte Auflage der Synonyma von 1564 die 6. Auflage des Proceß von 1562 benutzt; vgl. Haß (1986: 223231).

53 Sieben der 43 Fremdwort-Lemmata sind in Fraktur gesetzt, doch sollte dieser Befund (hier wie andernorts) nicht zu mechanisch als Indiz für die Einschätzung des Integrationsgrads der betreffenden Lexeme durch den Autor interpretiert werden. 


\section{Die Cortuntuiette Beatemifthe \\ Esort/ / aton anfange gemeldet/onb ben orfen Ennonp:} mis vertcutid t bito aufigelegt/fino bife.

Onberftid briber oen Buthfiab

2.

9y Drocak.

nippellixn.

apolect.

2 Lrefítirt.

\section{Bno dann ben Dein Bort/}

Xbgewinnen,

2abjoeuben

abrectang

abtbun

ainig werben

Xnbringen!

anlaicen

Anfagem

anwalofegen

Aufibaleens

4uffiboren!

auffidien/ Anfifdicten

zupgebent

2uศqun

zuperudentider

Xufiugy

apoe

Exception.
lurameneum It

2.

Xebing

Deoingen

Degeben'

Deredoen!

Berdfferw

Seffern/

2)eftetten

Euincirn.

Lauirn.

Cetion.

Abolim.

Perieim,

Callirn.

Anullirn.

Recafirn.

Cancellirn.

Tranfgirn.

Infinuarn.

Compromitrirn

SAuitirn,

Certificim,

$r$ Conftituirn.

LSubftiruirn.

$\{$ Sufpendirn.

Forum declinirn.

$\left\{\begin{array}{l}\text { Aufcultirn. } \\ \text { Collarionirn. }\end{array}\right.$

Relexirn.

Deliberirn.

Exhibirn

Edirn.

Elisirn.

Perimirn.

Specification.

Condition.

Proceftion.

Renuncïrn.

luftifieirn.

Denuncïrn,

Citirn.

Reformirn.

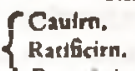

$\left\{\begin{array}{l}\text { Cautrn. } \\ \text { Raetficirn. }\end{array}\right.$
Qt

Ditten/

Surg/कaffer

Citation.

Confpiradon,

Codicill.

Commiflari.

Compafue.

Compromils.

Compulforial.

Credeniz.

Contum.sx.

Confifarn.

Citirn/

Clas:

Suppliclin

Causion.

s.

Cieation.

Conuention.

Reconuention.

Reconueruirn.

\&.

Examen.

Executorial.

Expens.

Eimrebel

\&inteben/

Ein|chreiben/

"Rinletzen/

Linoelicb.

4 ineerben

Entlesen:

Lrb

Ertommen/

Errlengen/

Er|banen/

Er|tgen

Erworm/

Fa.for.

Fardien.

Pifrue.

Fif́al.

Sarbringen

Stag'

Scyren
Sexception.

Uacernupition. Excipien.

Immatriculira. Refrituirn。

Peremptoric:

Exhnetedirn. Priuirn. Legieima.

Recognoldrn.

Prorogirn.

Comparirn.

CPruferibirn.

$\{$ Prafcriptio.

Vícapio.

8.

Repetia.

$\left\{\begin{array}{l}\text { Produrtor. } \\ \text { Reproducirn } \\ \text { Inqu fition. }\end{array}\right.$

Den Ferịi renun. म̈rn.

Abb. 1: Leonhard Schwartzenbach: Synonyma (Frankfurt am Main 1564), fol. 101a. 
(3.

Geferts

Geleit

Sidb gefdbiftemaden/

Grundoel

Eemaler

Beforfin

Begenteder

bersegy

binocrlegens

Inftrument.

Inuencirn.

Interefle.

Extimation.

Liels conteftation,

Lebigen/

SN.
Comites, Regalis

Commeatur. Remifr.

Qualificirn. Relegirn.

Fundament. Repreffalien. Spols. Xidten/

Legatum.

Replic. $\left\{\begin{array}{l}\text { Duplic. } \\ \text { Triplic. }\end{array}\right.$

ร.

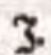

Duces,

Sequeftrim,

Symoney.

Syndicus.

Edakgen/

Sdomador

Exury

Estoictman!

Edoledt!

3.

Decerniran

9.

Tax.

Tyranney.

Tyrannifirn.

Ceftament/

$\left\{\begin{array}{l}\text { Abfoluirn. } \\ \text { Quirtirn. }\end{array}\right.$

Dergebers

2.

Codicill

Summarté ons de plano.

z.

Taxhe

Inturiea

Defentara

Arbicer.

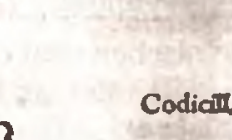

Alienim.

Corspentrm.

Dergleident

Admindilester

Dajitrang

Eratd

$\left\{\begin{array}{l}\text { Confinien. } \\ \text { Limiraneiprzpolit. }\end{array}\right.$

Derwerffer.

R.

Derbirgert/

Praferiprion.

SReculin.

$\{$ Reprobirs

Insercedira

Diterfosiben

Specificim

Tranfialom.

Exequira

Sulpendirn

radbfolgen/

reuswerung

Obligadon.

D.

Succedirn. Dollbringen

$\{$ Curator.

Dabalten!

Tutor

Dormonber/

Ongeborfans

Original.

p.

Damurang

Polfer.

Principal.

probirn.

Pfantor

protboedll

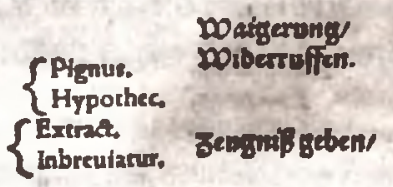

28.

Soniedur.

$\{$ Prefumpdion

Reduation.

Reuodirn.

8.

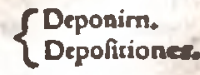

E RDE.

Abb. 2: Leonhard Schwartzenbach: Synonyma (Frankfurt am Main 1564), fol, 101 b. 
Göbler'schen Bezeichnung die darin angelegte ablehnende Haltung weiter, doch würdigt er mit seinem Register immerhin die Sonderstellung der Fremdwörter im Deutschen und trägt ihr lexikographisch Rechnung. Von hier aus ist der Schritt zum „ersten Fremdwörterbuch“ nicht mehr allzu groß.

Eine hochinteressante Weiterentwicklung erfährt die Redeweise von den korrumpierten lateinischen Wörtern schließlich ebenda, bei Simon Roth. In seinem Teutschen Dictionarius von 1571 bezeichnet er in einigen seiner Wortartikel das jeweils behandelte Fremdwort zwar, dem Wortgebrauch seines Lehrers Aventinus folgend, als "zerbrochens Lateinisch wort"54 (einmal sogar als "corrumpirts Lateinisch wort" ${ }^{45}$ ) bzw. als „zerbrochens Griechisch wort"56. Dann aber beschließt er, ganz ähnlich wie Schwartzenbach, das Wörterbuch ebenfalls mit einem (hier: vierseitigen) Glossar, das „korrumpierte“ Wörter auflistet (fol. R2a-R3b). In der Uberschrift heißt es: „Volgen etlich Bawren Latein / das ist / wie der gemain Mann die Lateinischen wort corrumpirt." (fol. R2a). Danach beginnt die Wortliste:

Für
Appellation,
Aufinuentirn,
Apostem,
Clauicordi,
Comum
Cantzler,
Commissar
Commission,
Dedit,
Defension,
Dialtea
(...)

papalo oder apelo.

Aufuentirn.

Pockstem.

Clauigur.

Comaun.

Catzler.

Pisari vl' milssari.

Miscampm oder campmis.

Derigit.

Refention. VVepsion. Refens schrifft.

Die alt ehe.

Hier wird klar: Roth geht es in diesem Glossar nicht, wie Schwartzenbach, einfach um gängige Fremdwörter, die er als verderbte Formen ihrer fremdsprachigen

54 Fol. C5a: Cantzler, ist ein zerbrochen Lateinisch wort / vnd haist auff guit Teutsch / der mann im gätter (...); K8a: Most, Ist ein zerbrochens Lateinisch wörtel / gantz Mustum, Ein newer vnuergerter wein (...); M2a: Pass, Ist ein zerbrochens Lateintsch wort / gantz passus, Ein schritt (...); M7a: Pfister. Ein zerbrochens Lateinisch wort / pistor / darein ein fnach art der Teutschen sprach gesetzt wirt (...).

55 Fol. N2a: Probst, Ein comumpirts Lateinisch wort / gantz præpositus, Ein furgesetzter (...)

56 Fol. L6b: Orgl, Ein zerbrochens Griechisch wörtel/gantz heist es Organon, Ein instrument. (...); 08b: Schem, Ein zerbrochens Griechisch wort / gantz Schema, Ein form / gstalt / kleydung (...). Statt „zerbrochen" oder "corrumpirt" tritt hler auch einmal „abgebrochen“ auf: fol. J4b: Kyrch, vom Griechischen wort Kyrios, Ein Herr / daruon Kyriaci / oder abgebrochen kyrch / das dem Herren zugehört (...). 
Etyma betrachtet, sondem vielmehr um korrumpierte - im Sinne von: entstellte, verstümmelte, verballhornte - Formen von solch „regulären“, lateinisch- oder griechischstämmigen, flexionsmorphologisch ins Deutsche integrierten Fremdwörtern - lexikalische Korruptelen, wie sie der ungebildete „gemain Mann“ gelegentlich unfreiwillig produziert. Solche Verballhornungen sind dem Lateinschulmeister Roth ein Gräuel, sie stellt er in diesem Glossar als „Bawren Latein“ an den Pranger. ${ }^{57}$ Gegen die ihnen zugrundeliegenden Fremdwörter dagegen hat Roth auch wenn er sie mit Aventinus gelegentlich als „zerbrochen" bezeichnet - nichts einzuwenden. Im Gegenteil: schon in der Vorrede zum Dictionarius gibt er sich als emphatischen Befürworter lateinischer Fremdwörter im Deutschen zu erkennen, die er als Bereicherung der deutschen Sprache und ihrer Ausdrucksmöglichkeiten willkommen heißt. ${ }^{58}$ Diese so segensreichen Fremdwörter empfindet Roth als wahrhaft "korrumpiert" eigentlich erst dann, wenn sie im Mund der einfachen "Bauern" monströse Gestalt annehmen.

Das ist eine ganz andere Einstellung als bei Aventinus oder Göbler, und keine, die in den folgenden Perioden immer wieder aufflammender Fremdwortfeindlichkeit besonders populär gewesen wäre. Trotzdem hat ausgerechnet Roth mit seinem kurzen Glossar „Bawren Latein" eine lexikographische Nebentradition ins Leben gerufen, die ihn um Jahrhunderte überdauert hat und erst im frühen 19.Jahrhundert zum Erliegen gekommen ist. Roth ist mithin nicht nur der „Erfinder" des deutschen Fremdwörterbuchs (oder Wörterbuchs der schweren Wörter), sondern auch eines lexikographischen Kuriosums: des Bauern-Lexikons.

Nicht wenige der von Roth mitgeteilten Verballhornungen von Fremdwörtern haben in den Ohren der sattelfesten Lateiner, also der Bildungselite, einen komischen Klang. Für Cantzler sagt der "Bauer", wie wir bereits gesehen haben, Catzler. Für Orgl sagt er Gurgl (fol. R2b). Aus Principal kann gleich ein ganzes

57 Allerdings gibt Roth auch im alphabetischen Wörterbuchteil gelegentlich solche korrumpierten Formen an: Armar, oder corrupte almar (...) (fol. C1b); Clistir, oder wie man corrupte spricht / Cristir (...) (C8b); Dom oder corrupte Thüm (...) (F3a); Prouision, oder comupt Prouison (...) (N5a); Pulpit, oder zerbrochen Pulpret (...) (N6a); vgl. Kremer (2013: 108) (ohne Dom und Pulpit). 58 Roth 1571, fol. A4a/b: „Nein du mein Lieber / Eben darumb / das die Teutsch sprach so zierlich worden / ist die Lateinisch vnnd Griechisch dester werder vnd fleissiger zuhalten vnd zu studiren / ohn welche solche zierligkeit nit recht kan verstanden werden / dieweil sie daraußjren vrsprung hat. [.. . ] Man sagt von den Latinern / Quid Latini haberent, si Græci non fuissent. Das ist / Was hetten wol die Lateiner / oder wie gar ein arme vnnd spröre sprach wer es / wenn die Griechen nit gewesen? Eben das mag man auch jetzundt von vns Teutschen sagen. Was hetten wir noch zumal für ein sprach / wann die Latein nit wär / welches dem Teutschen nit ein kleine zier gibt / Ich wil hie [... ] allein sagen unnd einem zuerwegen fuirgeben haben / was zier die Teutsch sprach allein auß den einzelligen wörtern / so auß dem latein eingemengt werden / bekommen habe / welche neben der zier auch grossen nutz mit bringen.“ 
Feuerwerk grotesker Korruptelen entstehen: Sprintzupalg, Parcifal, Runcifal, Paxifal oder gar Belzebock (fol. R3a). All diese himmelschreienden Entstellungen, als Zeugnisse gescheiterter Kommunikationsteilhabe durch bildungsferne Sprachbenutzer eigentlich nicht frei von einer gewissen Tragik, bieten Angehörigen der privilegierten Sprachelite, je nach persönlicher Veranlagung, durchaus Anlass zu selbstzufriedener Erheiterung. ${ }^{59}$ Vermutlich ist schon Roths kurzes Glossar von manchen so gelesen und goutiert worden. Zwar lässt Bernhard Heupold in seinen beiden kaum veränderten Nachdrucken des Dictionarius in den Jahren 1602 und $1620^{60}$ das Glossar mit dem Bauernlatein weg, doch im frühen 18. Jahrhundert wird diese Art der Korruptelenlexikographie wieder aufgegriffen.

1728 erscheint von einem Autor, der sich hinter dem Pseudonym Belemnon versteckt, ein Curiöses Bauern-Lexicon, in dem „Die meisten in unserer Teutschen Sprache vorkommende fremde Wörter erkläret- so dann, wie Ketzerlich solche von vielen ausgesprochen, angedeutet- und endlich bey jedem Wort eine lächerliche Bäuerisch- oder Jüdische Redens-Art beygefüget wird" ${ }^{61}$ Hier werden zu rund 800 Fremdwörtern rund 2.000 Abusivformen aufgeführt, deren irrtümliche Verwendung durch „Bauern" der Autor als "corrupte pronunciation“ (Vorr.: [7]) bezeichnet - es geht aber meist nicht um falsch ausgesprochene, sondern um falsch verstandene und völlig falsch verwendete Fremdwörter, jeweils belegt durch vorgeblich authentische Verwendungsbeispiele, die oft verdächtig komische Anekdoten oder (Herren-)Witze ergeben. ${ }^{62}$ Diese Art humoristischer Lexikographie falsch gebrauchter Fremdwörter findet dann im 18. Jahrhundert noch hier und da Wider-

59 Dass ihm der komische Nebeneffekt dieser Korruptelen bewusst ist, räumt Roth in seiner Vorrede ein (fol. A.7a): „Nun muß ich melden / das ich zu endt dises Dictionarij etliche cormupte wort / welche man Paurn latein haist / angehengt hab / Welches eins theils von nutz wegen / dann solche wort auch offt den Gelerten ein jrrung vnd nachsinnen machen / darneben aber auch zu eim possn vnd lächerlichem schwanck geschehen / damit man sehe / wie der gemein vngelert man / ein böser nachthur ist".

60 Bernhard Heupold: Teutsches DICTIONARIOLVM Welches außlegt vnnd erklärt etliche schwere vnbekande / Teutsche / Grichische / Lateinische / Hebraische / Welsche vnd Frantzösische / auch anderer Nation Wörter / so mit der weil in Teutsche Sprach kommen seind. Frankfurt: Egenolff 1602 (VD17 23:314328Z); Bernhard Heupold: DICTIONARIVM: Erklärend Allerley schwäre / vnbekandte / Teutsche / Griechische / Latinische / Hebraische / Italiänische / Frantzösische / auch anderer Nationen Wörter / so mit der weil in die Teutsche Spraach eingerissen / [...] auffs new vnd fleissigst zusammen getragen / vnd vmb viel verbessert. Basel: Genath 1620; vgl. Kremer (2013: 205-222).

61 Belemnon: Curiöses Bauem-LEXICON [...]. Denen Unwissenden zu wohlmeynenden Unterricht, andern aber zur Ergötzlichkeit zusammen getragen und zum Druck befördert Von BELEMNON. Freystatt 1728. Vgl. dazu Schmidt (2008).

62 Schmidt (2008: 8f.). 
hall ${ }^{63}$, um schließlich mit zwei Fremdwörterbüchern des frühen 19. Jahrhunderts auszuklingen. ${ }^{64}$ Auch wenn die Autoren der meisten dieser Wörterbücher von Simon Roth vielleicht nie etwas gehört haben, stehen ihre lexikographischen Werke mit ihrem Fokus auf korrumpierte Fremdwörter doch letztlich in der Tradition von dessen Glossar Bawren Latein im Dictionarius von 1571.

\section{7 lateinisch (griechisch, hebräisch usw.)}

Eine weitere Art schließlich, wie das 16. Jahrhundert Fremdwörter bezeichnet, besteht darin, sie nach ihrer Herkunftssprache zu benennen, als gehörten sie dieser nach wie vor an. Lateinischstämmige Fremdwörter werden dann also schlicht als lateinische Wörter bezeichnet, ungeachtet ihrer womöglich eingedeutschten Flexionsendungen, Orthographie oder Aussprache. Simon Roth verfährt so mehrfach, einmal in der bereits betrachteten Uberschrift seines Glossars mit Bawren Latein, wo er die Korruptelen von Fremdwörtern als korrumpierte Formen von lateinischen Wörtern bezeichnet (s. o.), dann aber auch in seiner Vorrede, wo er (fol. A5a/b) ausführt:

Doch gib ich hie nit für / verheiß auch nit / das man alle vnd jede Lateinische wörter / so im Teutschen bißher gebraucht seind worden / inn disem Dictionariolo finden werde / dann deren noch täglich mehr vnd mehr herfür kommen. Ich hab hie nur das maist vnd gebreuchlichest wöllen einfüren.

Auch in seinen Wortartikeln weicht Roth von der bei ihm häufigeren (und korrekteren) Ausdrucksweise, ein Fremdwort komme von einem bestimmten Etymon her (Muster: „Falck, von dem Lateinischen wort falco"; fol. G2a) nicht selten ab zugunsten der verkürzten Aussage, dass das Fremdwort ein Wort der Fremdsprache sei („Lai, Ein Griechisch wort / Einer auß dem gemeinen Volck“; fol. J5b).

Im Falle der Kleinen Wundartzney (s. o. 3.3) hat sich bereits gezeigt, dass das ursprüngliche Syntagma verlateinete vnd frembde wörtter in den ersten zehn Auf-

63 Nachdruck 1752 in leicht gekürzter Form in dem ebenfalls unter Pseudonym und mit fingiertem Druckort erschienenen Werk Ludwig Tölpels ganz funkel nagel neue BaurenMoral mit einem lächerlichen Wörterbuch vermehret und in das Teutsche übersetzt von Palato, Kamtschacka, darin 99-272 unter dem Titel Critisches Bauren-Wörterbuch (Schmidt 2008: 5).

64 Christian Anton August Slevogt: Alphabetisches Wörter-Buch besonders für Unstudierte: zur Erklärung der gewöhnlichsten in der deutschen Sprache vorkommenden fremden Wörter und Redensarten; nebst Beyspielen und Anecdoten. 4 Bände. Jena 1801; Eucharius Ferdinand Christian Oertel: Gemeinnüzziges Wörterbuch zur Erklänung und Verteutschung der im gemeinen Leben vorkommenden fremden Ausdrükke. Dritte, sehr verbesserte und vermehrte Auflage, Ansbach 1816 (EA 1804, 2. Aufl. 1806, weitere Ausg. 1826, 1831, 1840). 
lagen (1528-1534) beibehalten, dann aber von 1552 an durch lateinische Wörter ersetzt wurde. Auch Ickelsamer betont in seiner Teutschen Grammatica an der bereits betrachteten Stelle (s. o. 3.1), dass die von ihm aufgezählten elf Beispiele für Fremdwörter "nit teütsche wort sein / sonder Lateinisch vnd Ghriechisch".

Gegen Ende des 16. Jahrhunderts erscheinen noch zwei weitere notarielle Handbücher, die das Göbler-Glossar mehr oder weniger unverändert übernehmen, aber nun von den Fremdwörtern nur noch als lateinischen Wörtern (ohne den Zusatz „im Deutschen korrumpiert") reden. Das erste ist das 1584 erschienene Manual, oder Handbüchlin der Notariatkunst von Anton Hatzger ${ }^{65}$; das darin enthaltene 30-seitige Fremdwortglossar (152-182) ist überschrieben:

Erclärung Etlicher Latinischen wörter / so in Gerichten / Contracten / Testamenten / vnnd allerhand Schrifften gemeinlich im brauch sind / den Notarijs zu wissen von nöten. (152)

Ganz ähnlich findet sich im anonym herausgebrachten Manuale Instrumentonum von $1589^{66} \mathrm{im}$ sehr langen, auf eine Inhaltsangabe des Bands hinauslaufenden Titel folgende Passage (mit Bezug auf das Fremdwortglossar am Ende des Bandes):

weitter angehenckt ein kurtze erklärung etlicher Lateinischer wörter vnd phrases / so jm Rechten vnd Gericht / so wol mündelich also schrifftlich / in gerichtlichen Recessen / Producten / auch allen andem Concepten / vnd Formularien sehr gebreuchig / allen Schreibern / Notarien / Procuratorn / auch Gerichts vnd dergleichen Amptspersonen / zuwissen sehr notwendig (fol.)(1a).

Auf fol. 6a-7a gibt es zudem ein Verzeichnis von anderthalb Seiten für „etliche höfliche Teutsche vnd Lateinische Wörter / gleicher bedeutung " mit synonymen Doppel- und Mehrfachformeln, die jeweils mindestens ein Fremdwort enthalten (das somit ebenfalls als lateinisches Wort bezeichnet wird): „Inuestiert vnd belehnet worden“, „Reuociren und in Recht fordern möge“, „Hat statuirt vnd gebotten“, "Contract vnd handlungen“, „Resoluirung / erörterung vnd erledigung“ usw. Das Fremdwortglossar am Bandende schließlich umfasst zwölf Seiten und beginnt fol. 58a mit folgender Uberschrift (einer gekürzten Fassung der Ankündigung im Titel, mit einer Wendung am Anfang, die wieder stärker an Göbler erinnert):

Sondere vnd eigene Wörter / so im Rechten vad Gericht / in Gerichtlichen Producten / vnd allen andern Concepten vnd Formularien sehr gebraucht werden / wie die im Latein vnd

65 Anton Hatzger: MANVAL, Oder Handbuichlin der Notariatkunst. Speyer: Bernhard Dalbin 1584 (VD16 2V 7452).

66 (anonym): MANVALE INSTRVMENTORVM, Allerley Offenlicher vnd Privat Instrumenten Brief- Vnd schrifften (...). Frankfurt am Main: Martin Lechler in Verlegung Christian Egenolphs Erben 1589 (VD16 M 671 / N 1865). 
Teutschen zu verstehen seyn / allen Schreibern / Notarien / Procuratorn vnd andern Gerichtspersonen / zuwissen nötig.

Es folgen, weitgehend unverändert, die ersten beiden Teile des Göbler-Glossars, der dritte Teil aber mit den Verben zeigt etliche Neuerungen: er ist strikt alphabetisch sortiert, enthält nun neben Verben auch Substantive, Adverbien und Syntagmen, hat deutlich kürzere Interpretamente und beginnt mit einer neuen Uberschrift, die den meisten dieser Änderungen Rechnung trägt (fol. 60a):

Vnd dann folgen andere Wörter / vnd zum theil auch gantze Phrases, so vor Gericht in denselben einkommnen Producten vnnd sonsten andern Schrifften zu reden vnd zu schreiben sehr gebräuchig / vnd derohalben zu wissen von nöhten / nach dem Alphabet verzeichnet.

Nach wie vor sind nur die Verben mit ihren -i(e)r(e)n-Endungen eingedeutscht und als Fremdwörter im heutigen Sinne anzusprechen, die übrigen Wortarten und die Syntagmen sind durchweg lateinisch; das ganze Glossar ist somit eine Mischung aus Fremdwörterbuch und zweisprachigem (lateinisch-deutschem) Wörterbuch. Die Uberschrift verzichtet denn auch auf irgendeine genauere Bezeichnung des behandelten Wortschatzes.

Ein letzter Beleg aus Ickelsamers Teutscher Grammatica von 1534 (s. 0. 3.1) soll diese Sammlung von Textstellen beschließen; er gehört vielleicht als (vereinzelter?) Sonderfall zu diesem Typ von Fremdwortbezeichnungen dazu. Ickelsamer beschäftigt sich auf fol. Dla mit der Worttrennung und bemerkt über das Fremdwort Cistern ,Zisterne': „Also auch das lateinisch teütsch wort / Cistern / lautet lieblicher also getailt / Ci-stern". Wie hier die Aufeinanderfolge von lateinisch und deutsch gemeint ist, wird nicht restlos klar: ist es die additive Aufzählung der sprachlichen Bestandteile dieses Worts, die heute am besten durch eine Schreibung mit Bindestrich wiedergegeben würde, oder stehen die beiden Sprachbezeichnungen einfach unverbunden nebeneinander, weil sich auch Ickelsamer nicht entscheiden konnte, ob er dieses Wort als lateinisches oder deutsches ansprechen soll?

\section{Fazit und Ausblick}

Geht man die oben präsentierte Sammlung von Textstellen, in denen von Autoren des 16. Jahrhunderts Fremdwörter bezeichnet werden (eine, wie gesagt, unvollständige und vorläufige Sammlung) Stück für Stück durch, ergibt sich der Eindruck eines onomasiologischen Felds, in dem noch vieles in Bewegung und unfest ist. Im Zentrum stehen einige Ausdrücke, die öfters auftauchen, ohne dass ei- 
ner die anderen schon deutlich überwiegen würde. An der Peripherie finden sich selten oder nur einmal gebrauchte Wendungen, die zum Teil noch im 16. Jahrhundert wieder verschwinden (verlateinete Wörter), zum Teil länger überdauern, aber auch in späteren Jahrhunderten (soweit sich das bei einem noch flüchtigen Blick auf diese bereits sagen lässt) selten bleiben: schwere Wörter, unbekannte Wörter, ausländische Wörter (erst an der Wende vom 18. zum 19. Jahrhundert wieder häufiger belegt), und, oben nur gestreift, barbarische Wörter (oder auch beiläufig anfallende, oben nicht eigens besprochene Ausdrücke, die auf weitere Nebenaspekte von Fremdwörtern abheben, wie z. B. sonder und eigen). Interessant, weil eben besser belegt, sind die wenigen Syntagmen im Zentrum des Wortfelds. Vergröbernd und strenggenommen unzutreffend, dabei aber doch relativ verbreitet sind die Wendungen, die statt des Fremdworts eigentlich sein Etymon bezeichnen, wie lateinisches Wort, griechisches Wort usw. Das (aus heutiger Sicht) zukunftsträchtigste Syntagma fremde Wörter erscheint schon relativ häufig, aber noch nicht so oft, wie man vielleicht hätte erwarten können, zumal es an vielen Stellen mehrdeutig bleibt und nur selten wirklich mit letzter Sicherheit Fremdwörter bezeichnet. Korrumpierte lateinische Wörter schließlich ist ein Syntagma, dessen Verwendungsgeschichte weitgehend aufs 16 . Jahrhundert beschränkt geblieben zu sein scheint. ${ }^{67}$ Als Bezeichnung für Fremdwörter ist es offenbar in den dreißiger Jahren entstanden und noch vor Ende des Jahrhunderts wieder ausgegangen, doch in diesen fünfzig, sechzig Jahren ist es relativ häufig nachzuweisen. Es ist der wohl am negativsten wertende Ausdruck von allen, die oben betrachtet wurden, und man könnte sich darüber verwundern, dass er im viel fremdwortkritischeren 17. Jahrhundert von sprachpuristischer Seite nicht weiterverwendet wurde. Offenbar kam hier ein bezeichnender Perspektivenwechsel zum Tragen, der den „mitfühlenden“ Blick vom Lateinischen aufs Deutsche umlenkte: im 16. Jahrhundert konnten die humanistischen Lateiner im Fremdwort noch das verderbte lateinische Wort beklagen, hundert Jahre später wurden Fremdwörter (vor allem wenn sie in Massen auftraten und aus dem Französischen kamen) eher als verderblich für das Deutsche empfunden. Nur als Bezeichnung für verballhornte Fremdwörter

67 Einen späten, vom Anfang des 18. Jahrhunderts datierenden Nachhall dieser Bezeichnung für Fremdwörter stellt allenfalls das Syntagma „verstümmelte lateinische Wörter“ dar, das sich im Titel eines von Christian Juncker verfassten Nouvellen-Lexicon, einem in Christian Weises Traktat Curieuse Gedancken von den Nouvellen oder Zeitungen (Frankfurt, Leipzig 1703) enthaltenen selbständigen Fremdwörterbuch, findet: Curieuses Nouvellen-LEXICON, Oder Kurtze und deutliche Erklärung/ wo nicht aller/ jedoch der meisten und vornehmsten in denen Zeitungen vorkommenden und nicht jederman so gleich verständlicher Deutscher/Frantzösischer und Italiänischer/auch zum Theil verstümmelter Lateinischer Wörter und Redens-Arten/Deren Ubersetzung auf Begehren einiger Freunde/der Studierenden und Politischen Jugend zum Besten/auch in die Lateinische Sprache beygefüget worden (ebd. 607; das Fremdwörterbuch erstreckt sich im Weise-Traktat von 607-876). 
überlebte der Ausdruck korrumpiert noch für eine gewisse Zeit, was immerhin als Indiz für eine neue (und zaghafte?) Wertschätzung des Fremdworts interpretiert werden könnte. Doch waren - und blieben - die bekennenden Befürworter vom Schlage eines Simon Roth die seltene Ausnahme, und eine - wie man heute vielleicht sagen würde - ,Willkommenskultur" gegenüber fremden Wörtern wollte in den in sprachlichen Dingen tonangebenden Kreisen Deutschlands nie so recht aufkommen.

\section{Quellen}

(anonym): MANVALE INSTRVMENTORVM, Allerley Offenlicher vnd Privat instrumenten Brief- Vnd schrifften / so in hohen Fürstichen vnd Herm Hoff-Cantzeleyen / auch andern geringern Stände vnd Städte Schreibereyen / Consistorien / vnd dergleichen Amptsverwaltungen / [...] gefertigt vnd gestellet werden. [...] Frankfurt am Main: Martin Lechler in Verlegung Christian Egenolphs Erben 1589 (VD16 M 671 / N 1865).

(anonym): Notariat vnnd Teutsche Rhetoric. Frankfurt am Main: Christian Egenolff 1538 (VD16 N 1870). Weitere Ausgaben: Frankfurt am Main: Chr. Egenolffs Erben 1561 (VD16 N 1874), 1565 (VD16 N 1875), 1571 (VD16 ZV 20783 / N 1876), 1578 (VD16 N 1877).

[Agricola, Georg (angebl.):] Rechter Gebrauch der Alchimei / Mitt vil bißher verborgenen / nutzbaren vnnd lustigen Künsten (...] \ Der Alchimistischen verlateineten wörtter außlegung. [...] [Frankfurt am Main: Christian Egenolff d. A. 1531] (VD 16 R 492). Weitere Ausgaben: Frankfurt am Main um 1535 (VD16 R 494), 1540 (VD16 R 497), 1549 (VD16 R 498), 1566 (VD16 R 500) und 1574 (VD16 R 501).

[Aventinus:] Johannes Turmair's genannt Aventinus Bayerlsche Chronlk. Herausgegeben von Dr. Matthias Lexer. Erster Band. Erste Hälfte. München 1882 (Johannes Turmair's genannt Aventinus Sämmtliche Werke. Herausgegeben von der K. Akademle der Wissenschaften. Vierter Band. Erste Hälfte).

Belemnon: Curiöses Bauern-LEXICON, Worinnen Dle meisten in unserer Teutschen Sprache vorkomımsende fremde Wörter erkläret / so dann, wie Ketzerlich solche von vielen ausgesprochen, angedeutet / und endlich bey jedem Wort eine lächerliche Bäuerisch-oder Jüdlsche Redens-Art beygefüget wird. Denen Unwissenden zu wohlmeynenden Unterricht, andern aber zur Ergötzlichkeit zusammen getragen und zum Druck befördert Von BELEMNON. Freystatt 1728.

[Göbler, Justinus:] GErichtlicher Proceß / auß grund der Rechten vnd gemeyner Ubung / zum fleissigsten in drei theyl verfosset. Frankfurt am Main: Christian Egenolff [1536] (VD16 G 2296). Weitere Ausgaben: Frankfurt am Main: Christian Egenolff 1538, 1542, 1549, 1555, 1562, 1567, 1578 (VD16 G 2297-2303).

Hatzger, Anton: MANVAL, Oder Handbüchlin der Notariatkunst. Allen vnd ieden / sonderllch den angehenden jungen vnd vngeübten Notarien, Offenen Schreibern vnd Gerichtschreibern / sehr dienstlich vnnd nutzlich. Speyer: Bernhard Dalbin 1584 (VD16 ZV 7452).

Hedio, Caspar: Chronica der Altenn Christlichen kirchen auß Euseblo / Ruffino / Sozomeno / Theodoreto / Tertulliano / Justino / Cypriano / vnd Plinio / durch D. Caspar Hedlo verteutscht. Straßburg: Georg Ulrich 1530 (VD 16 E 4286). 
Heupold, Bernhard: Teutsches DICTIONARIOLVM Welches außlegt vnnd erklärt etliche schwere vnbekande / Teutsche / Grichische / Lateinische / Hebraische / Welsche vnd Frantzösische / auch anderer Nation Wörter / so mit der weil in Teutsche Sprach kommen seind / vnd offt mancherley Jrrung bringen [...]. Frankfurt: Egenolff 1602 (VD17 23:314328Z). Weitere Ausgabe: Bernhard Heupold: DICTIONARIVM: Erklärend Allerley schwäre / vnbekandte / Teutsche / Griechische / Latinische / Hebraische / Italiänische / Frantzösische / auch anderer Nationen Wörter / so mit der weil in die Teutsche Spraach eingerissen / vnd offt mancherley jrrung bringen [...]. Basel: Genath 1620.

ICkelsamer, Valentin: Teutsche Grammatica. Darauß ainer von jm selbs mag lesen lernen / mit allem dem / so zum Teätschen lesen [.. . zu zủ wissen gehört. [Augsburg, um 1535] (VD16 I 26).

[Lanfrank / Braunfels:] Kleine Wundartzney des hochberümptenn Lanfranci l...] / durch Othonem Brunfels verteutscht. [Augsburg: Heinrich Steiner 1528] (VD16 L. 245). Weitere Ausgaben: Straßburg: Christian Egenolph 1528 (VD16 L 247); Straßburg: Johann Schott 1528 (VD16 L. 248); Erfurt: Melchior Sachs 1529 (VD16 L 249); Zwickau: Gabriel Kantz 1529 (VD16 L 253); Erfurt: Melchior Sachs 1530 und 1534 (VD16 L 254 und L 255); Frankfurt am Main: Hermann Gulfferlch 1552 (VD16 L 256); Magdeburg: Johann Francke 1585 (VD16 L 260); Frankfurt am Main: Peter Kopf, Johannes Sauer 1594 (VD16 L 261).

Luther, Martin: AVszlegung der Epistelen vnd Euangelien / die nach brauch der kirchen gelesen werden durch den Aduent/vnd dannenthyn vom Christag biß vff den Sontag nach Epiphanie [... J. Basel 1522 (VD16 L 4551).

[Luther, Martin:] DAs neuw Testament recht grüntlich teutscht. Basel: Adam Petri 1523 (VD16 B 4325).

[Melanchthon, Philipp:] Verzaichnung vnd kurtzliche antzaigung in das Euangelium Joannis Philippi Melanchthons. [Augsburg: Grimm] 1524 (VD16 M 2485).

Oertel, Eucharius Ferdinand Christian: Gemeinnüzziges Wörterbuch zur Erklärung und Verteutschung der im gemeinen Leben vorkommenden fremden Ausdrukke, nach ihrer Rechtschreibung, Aussprache, Abstammung und Bedeutung aus den alten und neuen Sprachen erläutert. Ein tägliches Hälfsbuch für Beamte, Schullehrer, Kaufleute, Buchhändler, Künstler, Handwerker und Geschäftsmänner aus allen Klassen. Dritte, sehr verbesserte und vermehrte Auflage. Ansbach: Gassert 1816. Weitere Ausgaben: 1804, 1806, 1826, 1831, 1840.

Riederer, Friedrich: Spiegel der waren Rhetoric, VB M. Tulio C. Vnd andern getütscht. Freiburg im Breisgau: Riederer 1493 (GW M38173).

Roth, Simon: Ein Teutscher Dictionarius / das ist ein außleger schwerer / vnbekanter Teutscher /Griechischer / Lateinischer / Hebraischer / Wälscher vnd Frantzösischer / auch andrer Nationen wörter / so mit der weil inn Teutsche sprach kommen seind / vnd offt mancherley jrrung bringen. Augsburg: Manger 1571 (VD16 R 3265).

Schwartzenbach, Leonhard: Synonyma. Formular, Wie man ainerley rede vnd mainung / mit andern mehr worten / auff mancherley art vnd weise / zierlich reden / schreiben / vnd außsprechen sol. Frankfurt am Main: Feyerabend und Hüter 1564 (VD16 S 4677). Weitere Ausgaben: Nürnberg: Georg Merckel 1554 und 1556 (Claes 1977:118, Nr, 451 und 122, Nr. 466), Frankfurt am Main: Hieronymus Feyerabend 1571 (VD16 S 4678), ebd.: Sigmund Feyerabend 1580 (VD16 S 4679).

Slevogt, Christian Anton August: Alphabetisches Wörter-Buch besonders für Unstudierte: zur Erklärung der gewöhnlichsten in der deutschen Sprache vorkommenden fremden Wörter und Redensarten; nebst Beyspielen und Anecdoten. 4 Bände. Jena: Stahl 1801. 
Tannhäuser. Die Gedichte der Manessischen Handschrift. Mittelhochdeutsch / Neuhochdeutsch. Elnleitung, Edition, Textkommentar von Maria Grazia Cammarota. Obersetzungen von Jürgen Kühnel. Göppingen: Kümmerle 2009.

[Thomasin von Zerklære:] Der wälsche Gast des Thomasin von Zirclaria. Hrsg. von Heinrich Ruckert. Mit einer Einleitung und einem Register von Friedrich Neumann. Berlin 1965. Weise, Christian: Curieuse Gedancken von den NOUVELLEN oder Zeitungen / Denen / ausser der Elnleitung / wie man Nouvellen mit Nutzen lesen solle / annoch beygefugt sind / Der Kern der Zeitungen vom Jahr 1660. bis 1702. Eine kurtzgefaste Geographie, Eine Compendieuse Genealogie aller in Europa reglerenden hohen Häuser / und dann Ein sehr dienliches Zeitungs-Lexicon, also verfasset von M. C. I. Frankfurt und Leipzig: Pfotenhauer 1703 (VD18 10354441).

Wernher der Gartenære: Helmbrecht. Hrsg. von Friedrich Panzer. 8., neubearbeitete Auflage besorgt von Kurt Ruh (Altdeutsche Textbibliothek; Nr. 11). Tubingen: Niemeyer 1968.

\section{Literatur}

Bußmann, Hadumod (21990): Lexikon der Sprachwissenschaft (Kröners Taschenausgabe; 452). Stuttgart: Kröner.

Claes, Franz (1977): Bibliographisches Verzeichnis der deutschen Vokabulare und Wörterbü. cher, gedruckt bis 1600. Hildesheim, New York: Georg Olms.

Deutsches Wörterbuch von Jacob Grimm und Wilhelm Grimm (hier: 1878; 1956). Leipzig: Hirzel.

Deutsches Wörterbuch von Jacob Grimm und Wilhelm Grimm. Neubearbeitung (2006). Hrsg. von der Berlin-Brandenburgischen Akademie der Wissenschaften und der Akademie der Wissenschaften zu Göttingen. 9. Band, F-Fux. Stuttgart: Hirzel.

Duden Fremdwörterbuch ( 51990$)$. Bearbeitet vom Wissenschaftlichen Rat der Dudenredaktion. Duden Band 5. Mannheim et al.: Dudenverlag.

Duden. Das große Fremdwörterbuch. Herkunft und Bedeutung der Fremdwörter (2000). Herausgegeben und bearbeitet vom Wissenschaftlichen Rat der Dudenredaktlon. Mannheim et al.: Dudenverlag.

Duden Fremdwörterbuch ( $\left.{ }^{7} 2001\right)$. Herausgegeben von der Dudenredaktion. Duden Band 5. Mannheim et al.: Dudenverlag.

Eisenberg, Peter (2012): Das Fremdwort im Deutschen. Berlin, New York: De Gruyter.

GW: Gesamtkatalog der Wiegendrucke. Hrsg. von der Kommission für den Gesamtkatalog der Wiegendrucke. Bd. I-VIII Lfg.1, Leipzig: Anton Hiersemann 1925-1940; Bd.VIIIff. hrsg. von der Deutschen Staatsbibliothek zu Berlin. Stuttgart, Berlin, New York 1972ff. Benutzt über die Datenbank Gesamtkatalog der Wiegendrucke (URL: https:

//www.gesamtkatalogderwiegendrucke.de/ [23.3.2020])

Haß, Ulrike (1986): Leonhard Schwartzenbachs "Synonyma". Beschreibung und Nachdruck der Ausgabe Frankfurt 1564. Lexikographie und Textsortenzusammenhänge im Fruhneuhochdeutschen (Lexicographica. Series Maior; 11). Tubingen: Max Niemeyer.

Heier, Anke (2012): Deutsche Fremdwortlexikografie zwischen 1800 und 2007. Zur metasprachiichen und lexikografischen Behandlung äußeren Lehnguts in Sprachkontaktwörterbüchern des Deutschen (Lexicographica. Series Maior; 142). Berlin, Boston; De Gruyter. 
Jones, William Jervis (1995): Sprachhelden und Sprachverderber. Dokumente zur Erforschung des Fremdwortpurismus im Deutschen (1478-1750). Berlin, New York: De Gruyter.

Kirkness, Alan (1975): Zur Sprachreinigung im Deutschen 1789-1871. Eine historische Dokumentation (Forschungsberichte des Instituts für deutsche Sprache Mannheim; 26). Tübingen: Gunter Narr.

Kirkness, Alan (1976): Zur Lexikologie und Lexikographie des Fremdworts. In Probleme der Lexikologie und Lexikographie. Jahrbuch 1975 des Instituts für deutsche Sprache (Sprache der Gegenwart 39), 226-241. Düsseldorf: Schwann.

Kluge, Friedrich ( ${ }^{1921): ~ E t y m o l o g i s c h e s ~ W o ̈ r t e r b u c h ~ d e r ~ d e u t s c h e n ~ S p r a c h e . ~ B e r l i n ~ u n d ~ L e i p-~}$ zig: Vereinigung wissenschaftlicher Verleger De Gruyter.

Kluge, Friedrich (21 1975): Etymologisches Wörterbuch der deutschen Sprache. Bearbeitet von Walther Mitzka, Berlin, New York: De Gruyter.

Kremer, Anette (2011): Simon Roths Ein Teutscher Dictionarius zwischen Fremdwörterbuch und hard word dictionary. Sprachwissenschaft 36 (2/3), 197-223.

Kremer, Anette (2013): Die Anfänge der deutschen Fremdwortlexikographie. Metalexikographische Untersuchungen zu Simon Roths Ein Teutscher Dictionarius (1571). Heidelberg: Winter.

Lexer, Matthias (1878): Mittelhochdeutsches Handwörterbuch. Dritter Band. Leipzig: Hirzel.

Müller, Peter 0. (2001): Deutsche Lexikographie des 16. Jahrhunderts. Konzeptionen und Funktionen frühneuzeltlicher Wörterbücher. Tübingen: Max Niemeyer.

Paul, Hermann (\$1966): Deutsches Wörterbuch. Bearbeitet von Werner Betz. Tübingen: Max Niemeyer.

Paul, Hermann ( ${ }^{1992): ~ D e u t s c h e s ~ W o ̈ r t e r b u c h . ~ B e a r b e i t e t ~ v o n ~ H e l m u t ~ H e n n e ~ u n d ~ G e o r g ~ O b j a r-~}$ tel unter Mitarbelt von Heidrun Kämper-Jensen. Tubingen: Max Niemeyer.

Paul, Hermann ( ${ }^{10}$ 2002): Deutsches Wörterbuch. Bedeutungsgeschichte und Aufbau unseres Wortschatzes. Bearbeitet von Helmut Henne, Heidrun Kämper, Georg Objartel. Tübingen: Max Niemeyer.

Polenz, Peter von (1999): Deutsche Sprachgeschichte vom Spätmittelalter bis zur Gegenwart. Band III: 19. und 20. Jahrhundert. Berlin, New York: De Gruyter.

Róssing-Hager, Monika (1992): „Küchenlatein" und Sprachpurismus im frühen 16. Jahrhundert. Zum Stellenwert der "Latinismen" in frühneuhochdeutscher Prosa. In Nikolaus Henkel \& Nigel F. Palmer (Hrsg.), Latein und Volkssprache im deutschen Mittelalter 1100-1500. Regensburger Colloquium 1988, 360-386. Tubingen: Max Niemeyer.

Schmldt, Herbert (2008): Belemnons Curiöses Bauern-LEXICON (1728): Ein Wörterbuch des falschen Fremdwortgebrauchs im frühen 18. Jahrhundert. Lexeminventar und Text. In Thomas Gloning, Iris Bons \& Dennis Kaltwasser (Hrsg.), Fest-Platte für Gerd Fritz. Gießen (URL: http://www.festschrift-gerd-fritz.de/files/schmidt_2008_belemnons-curioeses-bauernlexikon.pdf [23.3.2020]).

VD16: Verzeichnis der Im deutschen Sprachbereich erschienenen Drucke des 16. Jahrhunderts. Hrsg. von der Bayerischen Staatsbibllothek in München in Verbindung mit der Herzog August Bibliothek in Wolfenbüttel. I. Abteilung. Stuttgart 1983ff. Online-Datenbank (URL: https://www.bsb-muenchen.de/sammlungen/historische-drucke/recherche/vd-16/ [23.3.2020]).

VD17: Verzeichnis der im deutschen Sprachbereich erschienenen Drucke des 17. Jahrhunderts. Online-Datenbank (URL: http://www.vd17.de/ [23.3.2020]). 
VD18: Verzeichnis der im deutschen Sprachroum erschienenen Drucke des 18. Jahrhunderts. Online-Datenbank (URL: https://g50.gbv.de/DB=1.65/SET=1/TTL=1/START_WELCOME [23.3.2020]).

Weigand, Friedrich Ludwig Karl ( $\left.{ }^{5} 1909\right)$ : Deutsches Wörterbuch. Nach des Verfassers Tode vollständig neu bearbeitet von Karl von Bahder, Herman Hirt \& Karl Kant. Hrsg. von Herman Hirt. Erster Band (A bis K). Gießen: Alfred Töpelmann.

Wiegand, Herbert Ernst (2001): Fremdwörterbücher und Sprachwirklichkeit. In Gerhard Stickel (Hrsg.), Neues und Fremdes im deutschen Wortschatz. Aktueller lexikalischer Wandel (Institut für Deutsche Sprache. Jahrbuch 2000), 59-88. Berlin, New York: De Gruyter. 